University of Nebraska - Lincoln

DigitalCommons@University of Nebraska - Lincoln

1977

\title{
Absolute Doubly Differential Cross Sections for Ejection of Secondary Electrons from Gases by Electron Impact. I. 100- and 200-eV Electrons on Helium
}

\author{
M. Eugene Rudd \\ University of Nebraska - Lincoln, erudd@unl.edu \\ R. D. DuBois \\ University of Nebraska - Lincoln
}

Follow this and additional works at: https://digitalcommons.unl.edu/physicsrudd

Part of the Physics Commons

Rudd, M. Eugene and DuBois, R. D., "Absolute Doubly Differential Cross Sections for Ejection of Secondary Electrons from Gases by Electron Impact. I. 100- and 200-eV Electrons on Helium" (1977). M. Eugene Rudd Publications. 49.

https://digitalcommons.unl.edu/physicsrudd/49

This Article is brought to you for free and open access by the Research Papers in Physics and Astronomy at DigitalCommons@University of Nebraska - Lincoln. It has been accepted for inclusion in M. Eugene Rudd Publications by an authorized administrator of DigitalCommons@University of Nebraska - Lincoln. 


\title{
Absolute doubly differential cross sections for ejection of secondary electrons from gases by electron impact. I. 100- and 200-eV electrons on helium
}

\author{
M. E. Rudd and R. D. DuBois * \\ Behlen Laboratory of Physics, University of Nebraska, Lincoln, Nebraska 68588
}

Received 8 March 1977

We have measured absolute values of the cross sections for ejection of electrons from helium gas by $100-$ and $200-\mathrm{eV}$ electrons. These cross sections were measured for emission angles from $10^{\circ}$ to $150^{\circ}$ and for electron energies from $3 \mathrm{eV}$ to a value equal to the primary energy minus the ionization potential. The measurements were made using a static-gas target and an electrostatic analyzer. The resulting cross sections agree reasonably well with those of Opal, Peterson, and Beaty near $90^{\circ}$, but show the need for an adjustment of their angular distributions. Comparison with Born-approximation calculations of Manson et al. at $2 \mathrm{keV}$ indicate that the cross sections of Opal et al. should be divided by $0.53+0.47 \sin \theta$. A somewhat larger correction is needed to bring their data into agreement with the results of this experiment. The adjustment in the angular distributions also affects the cross sections integrated over all angles.

C1977 The American Physical Society

URL: http://link.aps.org/doi/10.1103/PhysRevA.16.26

DOI: 10.1103 /PhysRevA.16.26

* Present address: Joint Institute for Laboratory Astrophysics, Boulder, Colo. 80302. 


\title{
Absolute doubly differential cross sections for ejection of secondary electrons from gases by electron impact. I. 100- and 200-eV electrons on helium
}

\author{
M. E. Rudd and R. D. DuBois ${ }^{\dagger}$ \\ Behlen Laboratory of Physics, University of Nebraska, Lincoln, Nebraska 68588
}

(Received 8 March 1977)

\begin{abstract}
We have measured absolute values of the cross sections for ejection of electrons from helium gas by 100 and 200-eV electrons. These cross sections were measured for emission angles from $10^{\circ}$ to $150^{\circ}$ and for electron energies from $3 \mathrm{eV}$ to a value equal to the primary energy minus the ionization potential. The measurements were made using a static-gas target and an electrostatic analyzer. The resulting cross sections agree reasonably well with those of Opal, Peterson, and Beaty near $90^{\circ}$, but show the need for an adjustment of their angular distributions. Comparison with Born-approximation calculations of Manson et al. at $2 \mathrm{keV}$ indicate that the cross sections of Opal et al. should be divided by $0.53+0.47 \sin \theta$. A somewhat larger correction is needed to bring their data into agreement with the results of this experiment. The adjustment in the angular distributions also affects the cross sections integrated over all angles.
\end{abstract}

\section{INTRODUCTION}

When energetic electrons collide with atoms or molecules, one of the important processes which takes place is ionization. A detailed understanding of ionization is important in many fields such as astrophysics, upper-atmosphere, plasma, and radiation physics, and charged-particle detector design. If is also, of course, of intrinsic interest because of its fundamental nature.

Early experimental work on the subject was largely confined to measurements of the total ionization cross sections although $\mathrm{Mohr}$ and $\mathrm{Nicoll}^{1}$ and Goodrich ${ }^{2}$ made measurements of cross sections for ionization differential in both angle and energy of the ejected and scattered electrons. Goodrich presented absolute values of these doubly differential cross sections (DDCS) while Mohr and Nicoll's cross sections were relative only. Early theoretical work concentrated primarily on total cross sections.

The availability of doubly differential cross sections measured by Opal, Peterson, and Beaty ${ }^{3,4}$ (hereinafter denoted $\mathrm{OPB}$ ) over a wide range of primary energies and target gases about five years ago stimulated new theoretical work ${ }^{5-8}$ which gives promise of providing accurate values of these cross sections at least for the higher impact energies but so far only for helium. In the work of Manson et al. ${ }^{6}$ a discrepancy was noted between this theoretical angular distribution and OPB's which they attributed to experimental problems.

Kim and co-workers at Argonne have devised a clever method $^{8}$ by which the energy distributions of secondary electrons (integrated over all angles of ejection) may be compared with photoionization cross sections and other data to assess their accuracy and to make adjustments when necessary.
By this means they have noted that with some exceptions the OPB cross sections are quite accurate. However, this method of comparison only deals with the cross sections differential in ejected energy $\sigma(E)$ and has nothing to say about the accuracy of the angular distributions except as they affect the integral over the angle. We present data here which indicate the need for some adjustment of the angular distributions presented by OPB.

OPB did not measure absolute cross sections but normalized their data to an elastic cross section reported by K. G. Williams ${ }^{9}$ and to total crosssection data. More recently Oda ${ }^{10}$ has presented DDCS for 500-eV electrons on helium which were normalized to elastic scattering data of Bromberg. ${ }^{11}$ Sethuraman, Rees, and Gibson ${ }^{12}$ have reported on measurements of normalized DDCS for helium but the data have not yet been published. At our laboratory G. B. Crooks ${ }^{13}$ measured absolute values of DDCS for ejection of electrons from helium by $50-800-e V$ electrons but his measurements were troubled by poor collection and detection efficiencies for low-energy electrons. However, he was able to show with his measurements at higher energies that agreement was good with OPB's work provided the latter were adjusted by multiplying by $1 / \sin \theta$. Ehrhardt and coworker $\mathrm{s}^{14}$ have reported on angular and energy distributions of electrons from 25- to $260-\mathrm{eV}$ electron collisions with helium and give cross sections in a few cases.

After making some modifications of the Crooks apparatus we have retaken the DDCS data in helium at 100 and $200 \mathrm{eV}$. As with Crooks' work, the cross sections are absolute in that they are calculated directly from measured quantities and do not depend on the results of any other experiment or calculation from theory. Our measurements 
range in angle from $10^{\circ}-150^{\circ}$ and in secondary energy up to $E_{p}-I$ where $E_{p}$ is the primary electron energy and $I$ is the ionization potential of the target. The data of OPB stops at $\frac{1}{2} E_{p}$ or lower and therefore omits most of the scattered electron contribution. It is, of course, impossible to distinguish the scattered primaries from the secondary electrons except that most of the scattered ones appear at the higher energies. By measuring the full range of energies one can make a useful check on the data by integrating over all angles. The resulting graph of singly differential cross sections $\sigma(E)$ plotted vs $E$ should be symmetric about $E_{v}=\frac{1}{2}\left(E_{p}-I\right)$ provided that the collision leaves a singly charged helium ion in its ground state. The cross section for double ionization ${ }^{15}$ is $0.4 \%$ of that for single ionization at $200 \mathrm{eV}$ and smaller at $100 \mathrm{eV}$. Simultaneous ionization and excitation was studied by Moussa and deHeer ${ }^{16}$ who measured cross sections totalling about $1.5 \%$ of the single-ionization cross section. Since the probabilities of these two processes are small, little departure from symmetry is to be expected. Doubly excited states leading to autoionization will yield peaks in the energy distribution at 33-41 $\mathrm{eV}$ and corresponding energy loss peaks symmetrically placed about $\frac{1}{2}\left(E_{p}-I\right)$. The cross section for producing these states may be estimated from the data of Oda et al. ${ }^{17}$ to be about $0.5 \%$ of the total ionization cross section. Thus autoionization may be expected to contribute small peaks which would represent a departure from the smooth continuum but which would not change the symmetry.

\section{EXPERIMENTAL APPARATUS}

The apparatus used is almost identical to that described previously in connection with our elastic cross-section measurements..$^{18}$ A focused collimated electron beam is produced by a rotatable electron gun. A fixed parallel-plate electrostatic analyzer of energy resolution $0.35 \%$ accepts electrons from the scattering center within an acceptance angle of $0.6^{\circ}$. After analysis the electrons are detected by a channeltron. Helium gas at a pressure of about $1 \mathrm{~m}$ Torr is allowed to flow slowly through the scattering region and constitutes an essentially static gas target. The pressure is measured by a capacitance manometer the calibration of which was checked against a McLeod gauge. Magnetic fields are reduced to below $5 \mathrm{mG}$ by a magnetic shield just inside the vacuum chamber.

The modifications made since the Crooks experiment to improve the accuracy of the low-energy measurements were as follows: (1) Additional magnetic shielding was added to prevent field penetration through the pumping hole. (2) Slots were cut in the back plate and field straightener plates of the electrostatic analyzer to prevent the generation of secondary electrons from surfaces struck by electrons of higher energy than the analyzer is set to pass. (3) We coated the inside of the analyzer plates and the acceptance collimation system with carbon soot. Although these precautions reduced the effect of scattering from surfaces to the point where fewer than 1 in 2500 electrons entering the analyzer outside its pass band were detected, there was still a small spurious peak at an analyzer setting of 0.32 times the primary energy. (4) The primary beam was caught by a new Faraday cup designed for better containment of the electrons. Also provision was made to monitor the current reaching the shield outside the cup in order to get a measure of the primary beam current missed by the cup. For the energies reported here the beam loss was $0.5 \%$ or less.

We used no preacceleration or other electron optics before the analyzer, thus insuring straight line paths and an easily calculable collection geometry. The total absorption cross sections for electrons as a function of energy measured by Golden and Bandel ${ }^{19}$ and by Normand ${ }^{20}$ were used to correct for absorption of electrons by the target gas. This correction was applied both to the primary beam and to the secondaries. Background counts from the residual gas were taken for all data and subtracted from the counts taken with the target gas present after the absorption correction. The background count at secondary energies above $10 \mathrm{eV}$ was less than $10 \%$ of the total. We made the assumption that the residual counts were unchanged by the presence of the target gas. Since the mean free path of electrons was considerably larger than the dimensions of the scattering chamber we feel that this is a good assumption. Furthermore, the background correction is so small that even omitting it entirely would not change our results by very much.

Below $10 \mathrm{eV}$, however, the background correction increased and was as large as $30 \%$ at $E_{p}=200$ $\mathrm{eV}$ and $60 \%$ at $E_{p}=100 \mathrm{eV}$. Because of the uncertainties involved in these large corrections, our low-energy data are less reliable.

The efficiency of the detector was $0.84 \pm 0.08$ from 10 to $200 \mathrm{eV}$ as determined previously. ${ }^{18}$ Relatively large variations of channeltron voltage, discriminator setting, beam current, and target gas pressure were found to have little or no effect on the calculated values of the cross sections. In every case, counts were taken for a long enough time to insure that the statistical uncertainty in the count was less than $3 \%$. The overall uncertainty in the cross sections varies with angle as well as with 
primary and secondary energy but generally speaking, the relative values are uncertain by $15 \%$ and the absolute values by $20 \%$ above $10 \mathrm{eV}$.

\section{EXPERIMENTAL RESULTS}

Energy distributions of secondary electrons for selected angles are shown in Fig. 1 for $100-\mathrm{eV}$ primaries compared with data from other investigators. The relative data of Mohr and Nicoll were normalized to that of OPB at $90^{\circ}$ and $45-\mathrm{eV} \mathrm{sec-}$ ondary energy. The general agreement among the experiments is fair. The data of OPB tend to be lower than the others at $30^{\circ}$ and Goodrich's low at $90^{\circ}$ relative to the other data. The measurements of Sethuraman et al., not on the graph, tend to be quite close to those of OPB except at $30^{\circ}$ and $150^{\circ}$ where OPB's are lower.

In Fig. 2 angular distributions of secondaries

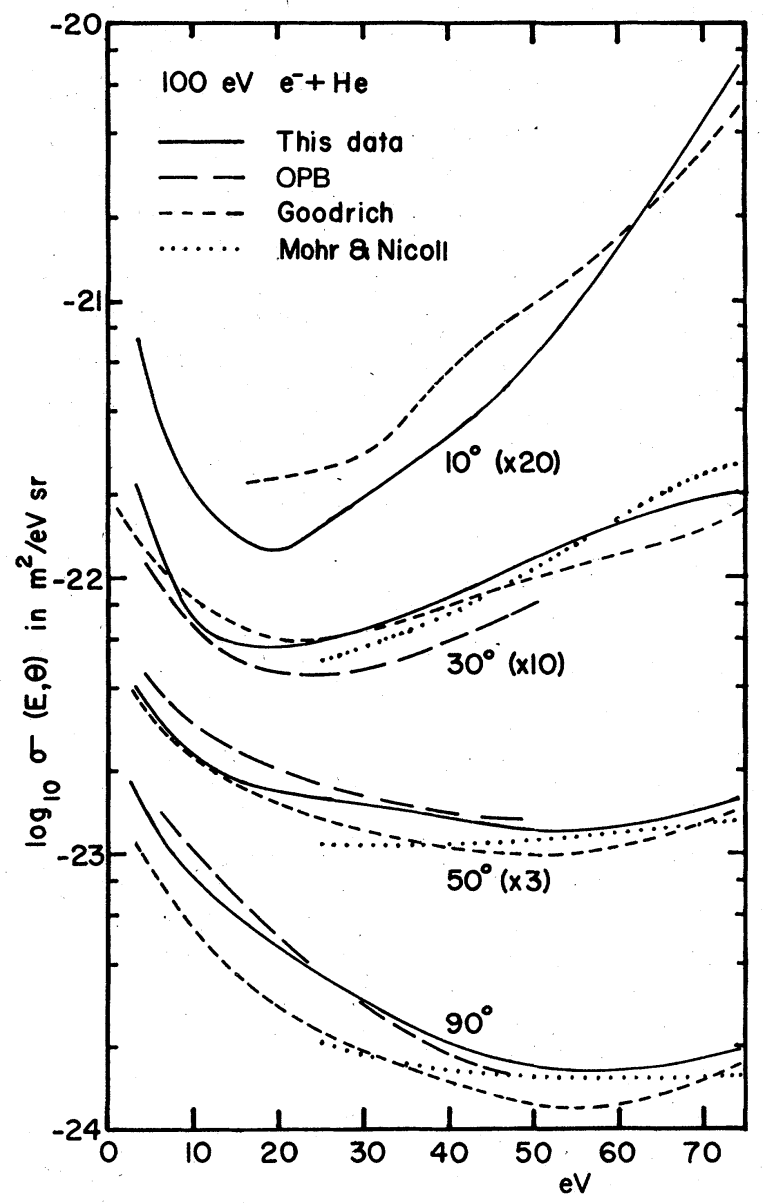

FIG. 1. Doubly differential cross sections for ejection of electrons from helium by $100-\mathrm{eV}$ electrons plotted vs ejected energy for four angles. Present data are compared to that of OPB (Ref. 3), Goodrich (Ref. 2), and Mohr and Nicoll (Ref. 1). from 200-eV collisions are shown at selected electron ejection energies. In our data there is a sharp rise in the cross sections below $20^{\circ}$ which is not present in the $100-\mathrm{eV}$ results. While this feature does not appear in the theoretical treatment of Manson et al..$^{6}$ it was seen by Crooks ${ }^{13}$ at the higher impact energies, by Oda and co-workers, ${ }^{10}$ and by Ehrhardt et al. ${ }^{14}$ Tahira and Oda ${ }^{10}$ applied the binary-encounter theory to the problem and found that the exchange term in that model led to a rise in the cross sections in the forward direction roughly corresponding to the experimental results. The exchange term becomes important when the momentum transfer approaches zero, that is, when the momentum of the ejected electron is nearly equal to that of the incident electron. Because of the binding energy, this condition can be more nearly fulfilled at higher impact energies. None of the other experiments have been done at the high incident energies and small angles needed to see this forward peak.

Also from Fig. 2 one notes a consistent disagreement between our data and that of OPB; namely, that relative to our measurements their cross sections drop off at the extreme angles. We contend that this indicates a need to correct their angular distributions.

In OPB's apparatus the target was in the form of

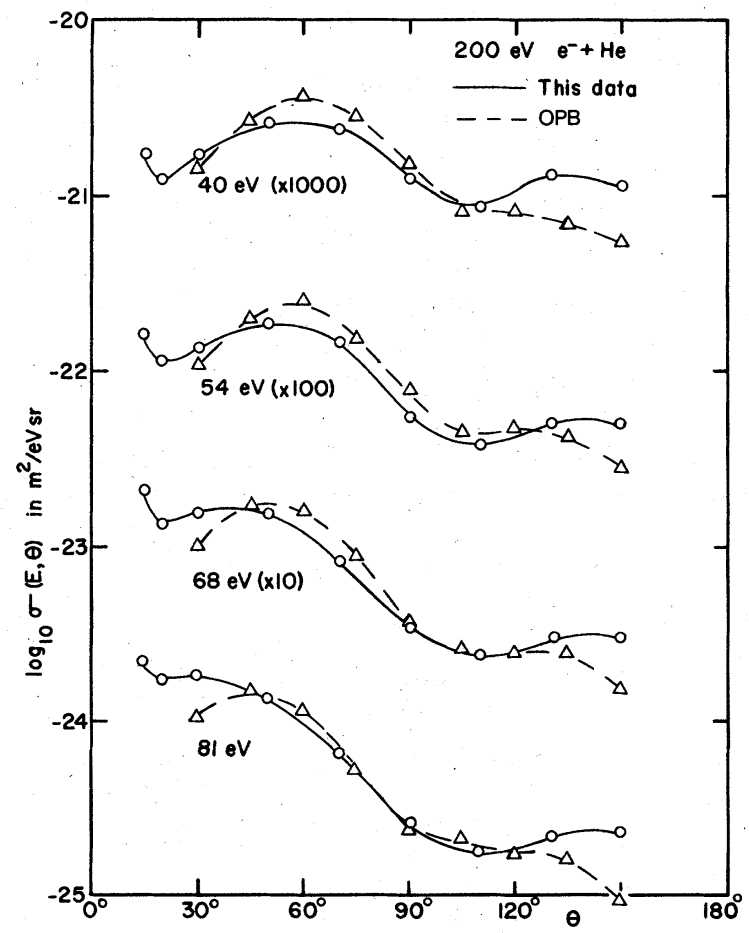

FIG. 2. Angular distributions of electrons ejected at various energies from helium by $200-\mathrm{eV}$ electrons. 
a broad beam of gas from a $6-\mathrm{mm}$-diam tube, 5 $\mathrm{mm}$ above the interaction region. Since the analyzer acceptance angle was large $\left(10^{\circ}-15^{\circ}\right)$ there is a question as to whether the target density was uniform over the entire length viewed by the analyzer, as assumed by OPB. The distance from the input aperture of the analyzer to the collision center was $5.75 \mathrm{~cm}$. At $30^{\circ}$ and $150^{\circ}$ an acceptance angle of $15^{\circ}$ would imply an electron beam length of over $30 \mathrm{~mm}$ viewed by the analyzer. Whether the atomic beam could spread out to uniformly cover that length of electron beam is very questionable: This matter was studied by the investigators themselves by comparing count rates at various angles using the atomic beam to those obtained by admitting the gas through a port out of the line of sight of the interaction region. The results of this comparison were given as Fig. 2.2 of Peterson's thesis. ${ }^{3}$ The ratio of these measurements does indeed show an angular dependence of the effective interaction length different from the $1 / \sin \theta$ assumed. The curve can be fitted by the relation $R=a+(1-a) \sin \theta$ with $a=0.53 \pm .25$ where $R$ is the ratio of the counts with the atomic beam to the corresponding counts with the static gas. This is the value of $a$ averaged over all angles with a $1-\sin \theta$ weighting factor. Unfortunately, OPB chose not to use the results of this check to correct their cross sections. Their assumption of a uniform gas density is equivalent to assuming $a=1.0$ in the above expression. If the gas beam had been smaller than the electron beam length viewed by their analyzer at $90^{\circ}$ the proper correction would have set $a=0$. It is our contention that OPB's cross sections would have been considerably more accurate if they had used the correction from their own experimental check as described above. This would mean that their reported cross sections should all be divided by $a+(1-a) \sin \theta$ with $a=0.53$. At $30^{\circ}$ and $150^{\circ}$, then, the correction would have been multiplication by a factor of 1.31. Since they give a $25 \%$ uncertainty in their angular distributions, the error bars do not quite cover this error. However, we believe that when the angular distributions are corrected, OPB's data are more accurate than their uncertainties indicate. OPB also indicated that an additional angular bias may occur in their experiment since their electron optics do not focus all parts of the interaction region equally well. Since the correction for the nonuniform gas density above brings the data of OPB into reasonably good agreement with other data to be examined, it is likely that the electron optical effect was small. Beaty ${ }^{4}$ has discussed their treatment of the angular distributions. In addition to the internal evidence concerning the need for a correction to OPB's angular distributions there is external evidence available from theory, from the present experiment, and from previously published experimental data.

Manson et al. ${ }^{6}$ have made Born-approximation calculations using Hartree-Fock wave functions. In their paper they pointed out the discrepancy between their calculated angular distributions and those of OPB and felt that the error was experimental. We have carried this comparison somewhat further. As the primary energy increases from 100 to $2000 \mathrm{eV}$, one would expect the results of the Born approximation to improve since it is basically a high-energy approximation. The agreement with OPB's experimental values is, indeed, found to improve steadily with increasing primary energy and at $2 \mathrm{keV}$ the angular distributions agree within $10 \%$ in the middle range of angles as shown in Fig. 3. This lends confidence to the use of the theoretical angular distributions to correct the data of OPB. In Fig. 4 we have plotted the ratio of OPB's data to the theoretical values of Manson, both normalized to $90^{\circ}$. These points represent averages over a range of secondary energies from 0 to $50 \mathrm{eV}$. At higher ejected energies the results are similar but not as reliable since they represent an interaction with a larger energy transfer while the Born approximation is most accurate when dealing with small perturbations. Also shown on Fig. 4 are the corresponding points derived from Fig. 2.2 of Peterson's thesis as described above. Note that the comparison with Manson's calcula-

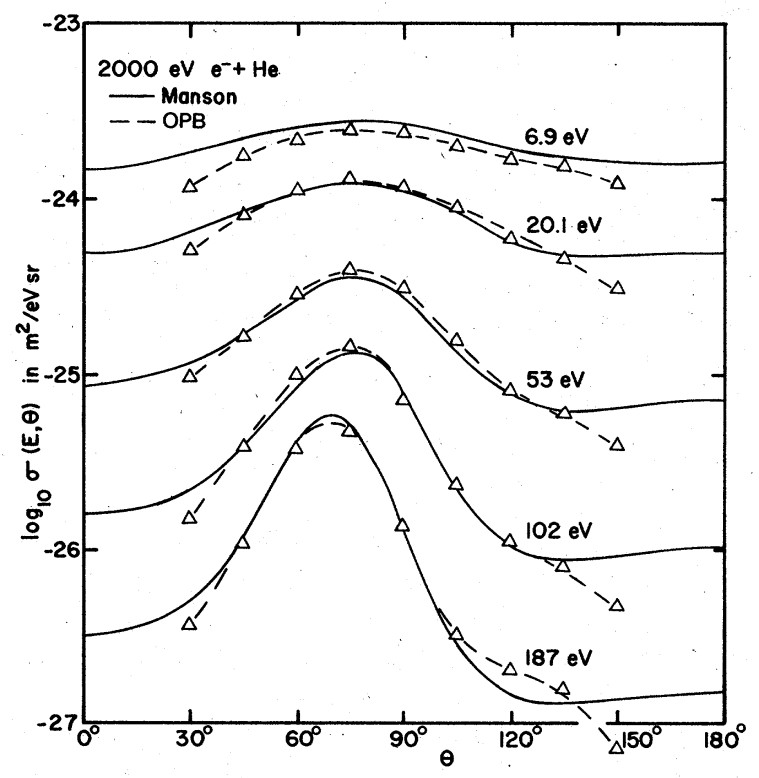

FIG. 3. Angular distributions of electrons ejected at various energies from helium by $2000-\mathrm{eV}$ electrons. Data of OPB (Ref. 3) are compared with theoretical calculations by Manson et al. (Ref. 6). 


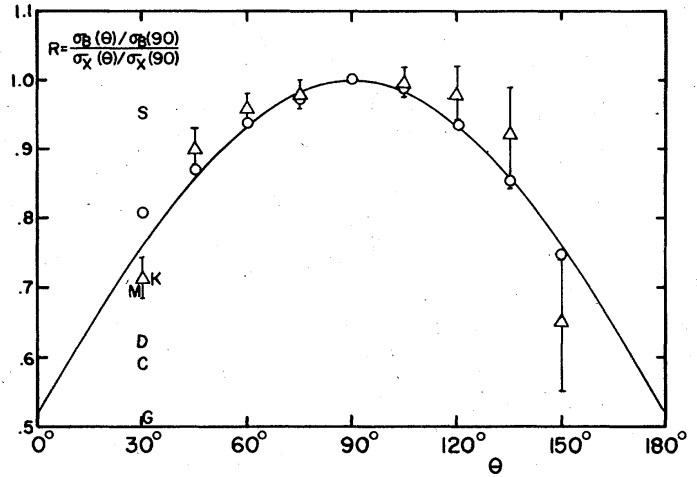

FIG. 4. Ratio of cross sections measured by OPB (Ref. 3 ) to those of other investigators, both normalized to $90^{\circ}$. Triangles are ratios to calculations by Manson et al. (Ref. 6) averaged over ejected electron energies. At $30^{\circ}$ ratio is to following: $D$, present data $100-200 \mathrm{eV} ; M$, Mohr and Nicoll 100-200 eV (Ref. 1); G, Goodrich 100 eV (Ref. 2); $C$, Crooks 50-200 eV (Ref. 13); $K$, Bell and Kingston 1 and $2 \mathrm{keV}$ (Ref. 5); $S$, Sethuraman et al. $50-500 \mathrm{eV}$ (Ref. 12). Circles indicate angular correction factors deduced from Fig. 2.2 of Peterson's thesis (Ref. 3 ). The line is a plot of $R=0.53+0.47 \sin \theta$.

tions indicates the need for a somewhat greater correction at $30^{\circ}$ and $150^{\circ}$ than Peterson's data but a similar correction at other angles. The best fit to the $2-\mathrm{keV}$ theoretical data of Manson requires a value of $a=0.53 \pm 0.18$. A comparison at $30^{\circ}$ only with the calculations of Bell and Kingston ${ }^{5}$ using somewhat different wave functions yields $a=0.42$ \pm 0.02 . The point labeled " $K$ " in Fig. 4 is from this data.

In our experiment we used a static gas and a narrow angular acceptance angle $\left(0.6^{\circ}\right.$ due to the analyzer and about $2^{\circ}-3^{\circ}$ primary beam spread) so there is no reason to suspect the standard $1 / \sin \theta$ correction to the effective beam path. Our previously published elastic cross-section measurements ${ }^{18}$ extended to angles as small as $2^{\circ}$ and excellent agreement was obtained with the work of Bromberg $^{11}$ and others. Even at that angle it was, found that the second-order geometrical corrections using the equations of Silverstein ${ }^{21}$ were less than $1 \%$.

In comparing our results with those of OPB, we have again taken the ratio of OPB's cross sections at $30^{\circ}$ to those at $90^{\circ}$ and plotted vs ejected electron energy. These curves were compared to similar ones from our present data as well as to those of other investigators. This comparison indicated that the constant $a$ in the above equation should be $0.24 \pm 0.12$ for our data, $0.18 \pm 0.10$ for Crooks, ${ }^{13} 0 \pm 0.18$ for Goodrich, ${ }^{2} 0.38 \pm 0.24$ for Mohr and Nicoll, ${ }^{1}$ and $0.90 \pm 0.06$ for Sethuraman et al. ${ }^{12}$ Calculations of $R$ from these values have been plotted in Fig. 4 for comparison. In every case except Sethuraman the experimental data indicate the need for an even smaller value of $\boldsymbol{a}$ than suggested by the internal evidence. While the data of Sethuraman, Rees, and Gibson agree much more closely with those of OPB, Rees had indicated by a private communication that their data are preliminary and are not believed to be reliable at $30^{\circ}$ and $150^{\circ}$. Because of the wide spread among the various experimental values of $a$ we suggest the use of the value $a=0.53$ obtained from the internal evidence and by comparison with Manson's calculations.

This correction affects the integrated cross section $\sigma(E)$ such that OPB's values must be multiplied by a factor $f$ where $1.00 \leq f \leq 1.89$ depending on the angular distribution. Any distribution linear in angle (including an isotropic distribution) yields a value of $f=1.11$. Figure 5 shows a plot of $\sin \theta \sigma(E, \theta)$ vs $\theta$ for a primary energy of $100 \mathrm{eV}$ and a secondary energy of $49 \mathrm{eV}$. The area under this curve is the cross section $\sigma(E)$. For the purpose of integration $O P B$ give a prescription for finding their cross sections at $15^{\circ}$ using a logarithmic extrapolation from the $30^{\circ}$ and $45^{\circ}$ points. We have used this method to obtain a cross section at $15^{\circ}$ as shown. At angles near $180^{\circ}$ the cross sections are usually small and discrepancies here make little difference to the integration: However, since much of the area is at small angles the correction to the $30^{\circ}$ and $45^{\circ}$ points can be important as seen on the graph. When the suggested correction is made on the OPB data, fairly close agreement with the other two curves is obtained.

The secondary energy chosen for this graph illustrates what is probably the worst case, however, and at lower energies OPB's cross sections in the middle range of angles are increasingly larger than ours so that the loss of area at the small angles is more than made up for by the greater area elsewhere. Thus Fig. 6 shows that their cross sections integrated over angle are

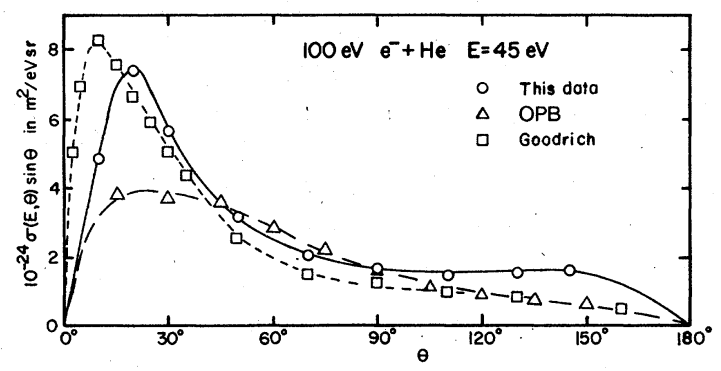

FIG. 5. Plot of $\sigma(E, \theta) \sin \theta$ vs $\theta$ for three sets of data at $E_{p}=100 \mathrm{eV}$ and $E=49 \mathrm{eV}$. OPB's point at $15^{\circ}$ is calculated by logarithmic extrapolation from the $30^{\circ}$ and $45^{\circ}$ points as used by those authors in integrating over angle. 


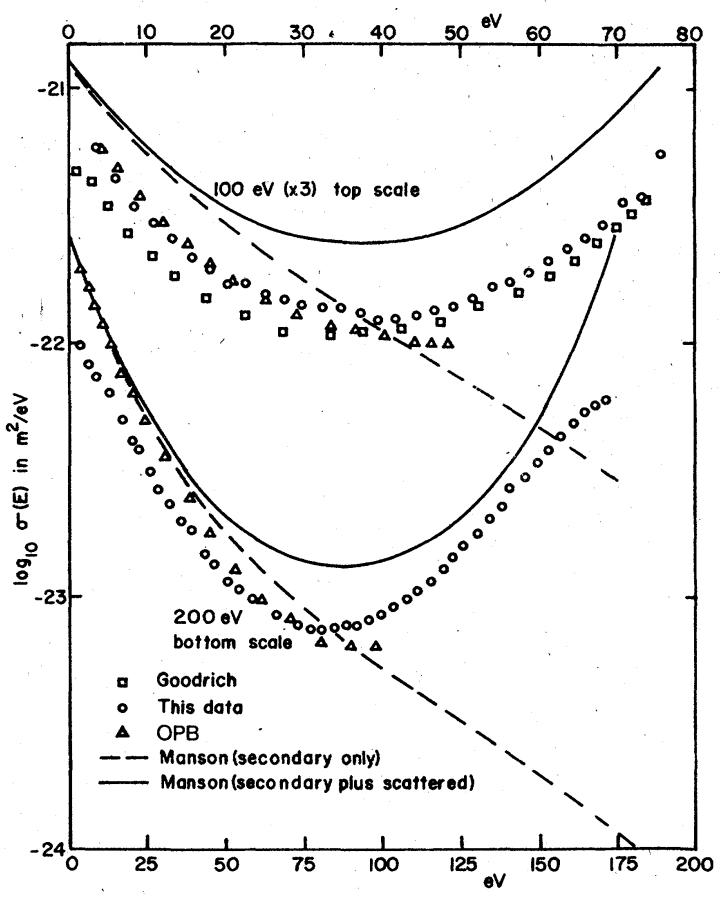

FIG. 6. Cross sections differential in ejected energy (integrated over all angles) vs energy for 100- and 200 $\mathrm{eV}$ electrons incident on helium. Experimental results compared to theoretical calculations.

larger than ours at most energies. Also plotted in that figure are the theoretical values of Manson et al. These have been "folded" to include the contribution due to scattered electrons. For each secondary electron of energy' $E$ there must be a scattered electron of energy $E_{p}-I-E$. If the scattered electron contribution is added to the secondary contribution the energy distribution is symmetrical about $E=\frac{1}{2}\left(E_{p}-I\right)$ as seen in Fig. 6. Our values, while somewhat below the theoretical curve, follow it quite well in shape over nearly the entire energy region. The departure from symmetry at high primary and secondary energies is due to the fact that there is a strong contribution of scattered electrons at very small angles. Our measurements down to $10^{\circ}$ or $15^{\circ}$ do not cover this peak well enough to give an accurate integral over angle for those parameters.

The data of OPB, especially at $200 \mathrm{eV}$, do not agree well in shape with theory and do not show the expected minimum at $87.7 \mathrm{eV}$. This may be due largely to the same reason; namely that at this energy there is a relatively large contribution to the cross section from the electrons directed in the forward direction. If OPB's $30^{\circ}$ point is too low this would depress the integrated cross section more here than elsewhere. It may also be because the calculations do not include exchange. At $100 \mathrm{eV}$ our data and OPB's agree well at all energies below about $30 \mathrm{eV}$. At higher energies our data show the required symmetry better than OPB's. The data of Goodrich are his original data before he multiplied by the factor 1.9 to plot in his Fig. 9. His results a re somewhat low but this is expected because of a nonlinearity in his McLeod gauge. Additionally, Goodrich evidently did not take account of absorption of electrons by the target gas and this would make his cross sections too small.

\section{CONCLUSIONS}

We have presented absolute cross-section data on the emission of electrons from impact of electrons on helium which agree well with the data of $O P B$ in the middle range of angles but which indicate the need for an adjustment of their data at large and small angles. The correction suggested is division of their data by $0.53+0.47 \sin \theta$. This adjustment results in a correction of about $11 \%$ in their integrated cross sections $\sigma(E)$ at small values of $E$ where the angular distribution is close to isotropic, but the correction is larger in cases where the distribution is peaked at forward angles.

\section{ACKNOWLEDGMENTS}

We wish to thank W. Peterson for useful comments, S. Manson for providing tables of his calculated cross sections, and M. Goodrich for sending graphs of his data.
*This was prepared with the support of National Science Foundation Grant No. PHY76-23348.

†Present address: Joint Institute for Laboratory Astrophysics, Boulder, Colo. 80302.

${ }^{1}$ C. B. O. Mohr and F. H. Nicoll, Proc. R. Soc. Lond. A 144,596 (1934).

${ }^{2}$ Max Goodrich, Phys. Rev. 52, 259 (1937).

${ }^{3}$ C. B. Opal, E. C. Beaty, and W. K. Peterson, At. Data 4, 209 (1972); W. K. Peterson, C. B. Opal, and E. C. Beaty, J. Phys. B $\underline{4}$, 1020 (1971); W. K. Peterson,
E. C. Beaty, and C. B. Opal, Phys. Rev. A $\underline{5}, 712$ (1972); C. B. Opal, W. K. Peterson, and E. C. Beaty, J. Chem. Phys. 55, 4100 (1971); and W. K. Peterson, thesis (University of Colorado, 1971) (unpublished).

${ }^{4}$ Earl C. Beaty, Radiat. Res. 64, 70 (1975).

${ }^{5}$ K. L. Bell and A. E. Kingston, J. Phys. B 8,2666 (1975).

${ }^{6}$ S. T. Manson, L. H. Toburen, D. H. Madison, and N. Stolterfoht, Phys. Rev. A 12, 60 (1975).

${ }^{7}$ Y. K. Kim and M. Inokuti, Phys. Rev. A 7, 1257 (1973). 
${ }^{8}$ Y. K. Kim, Radiat. Res. 61, 21 (1975); 64, 96 (1975); 64, 205 (1975); and Y. K. Kim and T. Noguchi, Int. J. Radiat. Phys. Chem. 7, 77 (1975).

${ }^{9}$ K. G. Williams, Proceedings of the Sixth International Conference on the Physics of Electronic and Atomic Collisions, Cambridge, Mass., 1969, edited by I. Amdur (MIT Press, Cambridge, Mass., 1969), pp. 731 and 735.

${ }^{10}$ N. Oda, Radiat. Res. 64,80 (1975); N. Oda, F. Nishimura, and S. Tahira, J. Phys. Soc. Jpn. 33, 462 (1972); and S. Tahira and N. Oda, ibid. 35, 582 (1973).

${ }^{11} \mathrm{~J}$. P. Bromberg, J. Chem. Phys. 50, 3906 (1969). ${ }^{12}$ S. K. Sethuraman, J. A. Rees, and J. R. Gibson, in Proceedings of the Eighth International Conference on the Physics of Electronic and Atomic Collisions, Belgrade, 1973; edited by B. C. Čobić and M. V. Kurepa (Institute of Physics, Belgrade, 1973), p. 316; and private communication.

${ }^{13} \mathrm{G}$. B. Crooks, thesis (University of Nebraska, 1972) (unpublished).

${ }^{14}$ H. Ehrhardt, K. H. Hesselbacher, K. Jung, M. Schulz, T. Tekaat, and K. Willman, Z. Phys. 244, 254 (1971).

${ }^{15}$ L. J. Kieffer and Gordon H. Dunn, Rev. Mod. Phys. 38, 1 (1966).

${ }^{16}$ H. R. Moustafa Moussa and F. J. deHeer, Physica 36, 646 (1967).

${ }^{17} \mathrm{~N}$. Oda, S. Tahira, and F. Nishimura, J. Phys. B $\underline{6}$, L309 (1973).

${ }^{18}$ R. D. DuBois and M. E. Rudd, J. Phys. B 8,1474 (1975); 9, 2657 (1976).

${ }^{19}$ D. E. Golden and H. W. Bandel, Phys. Rev. 149, 58 -(1966).

${ }^{20}$ C. E. Normand, Phys. Rev. 35, 1217 (1930).

${ }^{21}$ E. A. Silverstein, Nucl. Instrum. Meth. 4, 53 (1959). 